\title{
HALLAZGO DE UNA NUEVA COLUMNA MILIARIA PERTENECIENTE A LA VIA AUGUSTA
}

\author{
E. RUIZ NIETO \\ L. APARICIO SANCHEZ \\ F. GODOY DELGADO (*) \\ J. COSTA RAMOS (**)
}

\section{Resumen}

Mediante este artículo damos a conocer una nueva columna miliaria correspondiente a la Via Augusta y hallada en las inmediaciones del Polígono Industrial de La Torrecilla. Su cronología, basada en el análisis epigráfico, nos permite adjudicarla al emperador Nerón, en los primeros años de su mandato.

\section{Summary}

By means of this article we let you know about the discovery of a new milliary column which belonged to the Roman Via Augusta. It has been found in the environs of La Torrecilla industrial area close to Córdoba. Its chronology based upon epigraphic analysis allow us to date it in the early years of Nero mandate.

El conocimiento de las vías de comunicación en época romana es una de las bases fundamentales para entender cl complejo fenómeno de la romanización, puesto que constituye un medio material imprescindible para el acercamiento del mundo romano al indígena y de éste entre sí.

Probablemente, una de las vías más antiguas y más transitadas del imperio romano fue la Via Augusta (PALLI, 1985:47), cuyo antecedente más remoto lo constituyó cl camino que iba desde Italia al sur de la Península Ibérica, que la leyenda y la tradición atribuyo a Heracles.

(*) Museo Arqueológico Provincial de Córdoba.

${ }^{(* *)}$ Delegación Provincial de la Consejería de Cultura y Medio Ambiente de Córdoba. 
Así, la Vía Augusta $a_{\perp}$ la gran vía romana que partiendo de Roma llegaba hasta Cádiz, fue la ruta de entrada en la Península Ibérica durante los siglos de la dominación romana y paso obligado para los que desde el sureste de la Galia se dirigían por tierra al sur de Hispania.

Fundamental para el estudio de las vías son las fuentes epigráficas (ROLDAN, 1975) y, de ellas, un tipo muy específico, rico en ejemplares y muy importante en esta vía en particular, son los miliarios. Se han definido (MELCHOR, 1988: 29) como "indicadores de distancias, realizados en material duro (mármol, granito, caliza, arenisca, etc.) con forma de columna o cipo (forma de paralelepípedo). Su altura oscila entre 0’90 m. y 2’35 $\mathrm{m}$. y el diámetro del fuste puede variar entre 0’40 y 0’50 m. Eran colocados junto a las vías, presentando un campo epigráfico donde se indicaban las distancias en millas romanas"' (1).

Hasta el momento disponemos de un abundante número de publicaciones referentes a miliarios de la Vía Augusta en la provincia de Córdoba, basta recordar los estudios de Hübner, Franco, Romero de Torres, Silliers, Portillo y Melchor. Sirva, pues, esta aportación nuestra como un eslabón más en el conocimiento de la gran vía que comunicó por tierra a la Colonia Patricia con la capital del imperio.

El miliario que aquí presentamos apareció en el transcurso de las obras que la Empresa Nacional del Gas (ENAGAS) viene realizando desde hace algunos meses en la provincia de Córdoba, con el fin de dotarla de una red primaria de distribución de gas natural para uso industrial y doméstico.

Dichas obras han sido objeto en diversos puntos de su trazado de una Intervención Arqueológica de Urgencia autorizada por la Dirección General de Bienes Culturales de la Junta de Andalucía, financiada por la Empresa Nacional de Gas (ENAGAS), y cuyos directores han sido dos de los firmantes de este trabajo. No obstante el hallazgo de la columna miliaria tuvo lugar en una zona no sujeta a cautela arqueológica.

Fue el pasado 15 de octubre de 1991, a raíz de una llamada anónima recibida en la Delegación de la Consejería de Cultura y Medio Ambiente de la Junta de Andalucía en Córdoba, mediante la cual se notificó la aparición de una columna fragmentada en dos, durante las labores de apertura de zanja y soterramiento de la tubería del gas.

Una vez recibida la denuncia, nos personamos en el lugar D. Francisco Godoy Delgado, Director del Museo Arqueológico Provincial de Córdoba, D. Julio Costa Ramos, Oficial de Arqueología de la Delegación de Cultura y Medio Ambiente de la Junta de Andalucía en Córdoba y Dña. Laura Aparicio Sánchez y D. Eduardo Ruiz Nieto, Directores de los trabajos arqueológicos en el trazado del gasoducto, encontrándonos con la pieza aludida y pudiendo comprobar que uno de los fragmentos, el de mayor tamaño, presentaba algunas líneas de escritura.

(1) La milla romana equivale a una distancia actual de 1.481 metros. Para el caso de la Vía Augusta las distancias que marcan los miliarios de la Bética toman como punto de partida el Arco de Jano Augusto, situado en el lugar donde la Vía Augusta entra en la Bética. 
Efectuadas en el mismo día las oportunas gestiones por el Director del Museo Arqueológico Provincial de Córdoba, se procedió al traslado de la columna hasta las dependencias del Museo, donde quedó depositada y registrada con el número 30.892.

\section{Localización del hallazgo}

El afortunado hallazgo se produjo en la margen derecha de la carretera N-IV MadridCádiz, dirección Sevilla, en una isleta próxima a la actual calzada, integrada en una zona verde del Polígono Industrial de La Torrecilla, frente a la delegación en Córdoba de la casa Nissan-Ebro (Lámina I y Figura 1).

Sus coordenadas U.T.M. son: X: 342.500 , Y: 4.190 .300 , y Z: 115 , del mapa a escala 1:50.000, hoja núm. 16-37 (923) de Córdoba, editado por Serv. Geográfico del Ejército.

\section{Descripción de la columna miliaria}

La pieza en cuestión se fragmentó en dos al extraerse del subsuelo, a juzgar por el estado limpio de las fracturas, que al ser instalada se ha comprobado que casa perfectamente. Está labrada en mármol negro veteado conocido también como piedra de mina gris o caliza micrítica cámbrica procedente de la sierra de Córdoba, al igual que los expuestos en el patio de los naranjos de la Mezquita-Catedral (CIL.II, 4.701; CIL.II, 4.712; CIL.II, 4.716 y CIL.II, 6.208) y en el patio del Museo Diocesano de Córdoba (CIL.II, 4.721 y CIL.II, 4.731).

Tras unirse los dos fragmentos el resultado es una columna miliaria cuya parte superior finaliza en forma de tronco cono recto, alcanzando 1'14 m. de altura total. Un tercer fragmento, que no se llegó a descubrir hubiera completado toda la columna por su base, aunque este, pensamos con cierta reserva, no llevaría epigrafía. El diámetro en la parte inferior es de 0’49 m. y 0’45 m. en la superior, lo que resulta ser un cilindro con leve tendencia cónica, al igual que los miliarios CIL.II, 4.721 y 4.731 .

El texto epigráfico está formado por cuatro líneas de escritura. El tipo de letra empleado en la composición del texto es la capitalis actuaria, cuyos rasgos acusan el origen de las trazas a pincel (BATLLE, 1946) y que, con pequeñas modificaciones, se utiliza a lo largo de los dos primeros siglos del Imperio. En concreto, el modelo de escritura empleada, es característica de la época del emperador Claudio, con cierta perduración durante el mandato de Nerón (Figura 2).

La interpunción entre las palabras y las siglas está marcada por una suerte de pequeños triángulos propios de la capitalis actuaria, pero no siempre del mismo tamaño, y con uno de los vértices hacia abajo, salvo en algunos casos en que aparece levemente desviado, como puede apreciarse en el dibujo (2).

(2) Agradecemos a Dña. María Dolores Mata Luque, Delineante del Museo Arqueológico Provincial de Córdoba, su colaboración en la realización del dibujo y al Museo por facilitarnos la copia. 
El espacio entre líneas es variable, debido probablemente a la ausencia de guías. Entre las líneas 1 y 2 la distancia oscila de 3 a $2 \mathrm{~cm}$., entre las líneas 2 y 3 es de 2'5 y 3'5 cm. $y$ de la 3 a la 4 se reduce de 2 a $1 \mathrm{~cm}$.

En cuanto al tamaño de las letras existen unas variaciones que oscilan entre los $40 \mathrm{y}$ los $57 \mathrm{~mm}$.

Texto

NERO.CLAVDIVS.DIVI.CLAVDI.F
GERMANICI.CAESARIS.N.DIVI
AVG.AB.N.TI.CAESARIS.PRO.N.TRIB
POI. IMP. COS

Transcripción NERO CLAVDIVS DIVI CLAVDI F(ilius)

GERMANICI CAESARIS N(epos) DIVI

AVG(usti) ABN(epos) TI(berii) CAESARIS PRON(epos) TRIB(uniciae)

POI(estate) IMP(erator) $\mathrm{CON}(\mathrm{n}) \mathrm{S}(\mathrm{ul})$

Traducción Nerón Claudio hijo del Divino Claudio, nieto de Cesar Germánico, tataranieto del divino Augusto, biznieto de Tiberio Cesar, Tribunicia

Potestad, Imperio, Consulado.

\section{Conclusiones}

El texto de la columna miliaria hace referencia al emperador Nerón, cuyo reinado comprende desde el 54 al 68 d.C. Si examinamos las tablas cronológicas de los emperadores romanos (CAGNAT, 1964:187) la datación de la columna miliaria sería anterior al 13 de octubre del año 54, en que tiene lugar la renovación por primera vez de la Tribunicia Potestate.

A nuestro parecer hemos juzgado la palabra POI de la línea 4, como una T sin el trazo horizontal en lugar de un I como numeral, pues con frecuencia en ciertos textos (CAGNAT, 1964:22) la barra superior llega a desaparecer, confunciéndose con un I.

Llama también la atención, por lo anormal del caso, que la letra A de AUG en la línea 3, se haya trazado como una X. Este rasgo de arcaismo es más común cuando existen nexos de dos o más letras, tal como XX en lugar de AU.

$\mathrm{La}$ inexistencia en el texto de numerales (3) que indiquen la distancia en millas romanas con respecto a un punto concreto, así como la ausencia de referencias al tipo de obra, constructiva o reparadora, que se realiz6 en la vía, parece indicar que estos miliarios tuvieron otra función. Según Melchor Gil (MELCHOR, 1991:76) los miliarios “'también

(3) De existir algún numeral dudamos mucho que pudiera situarse en el lugar de la fractura. 
fueron utilizados como mero instrumento de propaganda imperial o para marcar la adhesión de determinadas ciudades a los nuevos emperadores".

La hipótesis anterior resulta plausible si mantenemos que la columna miliaria corresponde a los primeros días del gobiemo de Nerón en el año 54 d.C.

Otra circunstancia que viene a confirmar lo antes expuesto es la proximidad a Colonia Patricia de los miliarios que hacen referencia a Nerón, conocidos hasta el momento. Estos fueron descubiertos en las principales vías que entraban o partían de la capital de la Bética.

Existen otros dos miliarios de la época de Nerón, aparecidos en las proximidades de Córdoba, en los que el texto es idéntico, aunque su datación no se puede concretar por no figurar en ninguno de ellos los numerales correspondientes a los poderes otorgados.

-CIL.II, 4719: Miliario de Nerón, con un desarrollo del texto idéntico al que ahora se presenta. Ha sido fechado (MELCHOR, 1987:61) en el 68 d.C. por el Consulado V. Sin embargo nosotros pensamos que puede existir un error de transcripción por cuanto paralelamente al Consulado $\mathrm{V}$, deberían de figurar los numerales de los demás poderes o títulos conferidos al emperador.

Apareció en la Ermita de Nuestra Señora de Linares, junto a Córdoba, al pie de Sierra Morena. Trasladado en 1750 al lapidario de Villaceballos y posteriormente al Museo del Marqués de Loring en Málaga, hoy se halla en paradero desconocido.

Por el lugar en que apareció, es muy probable que se trate de un miliario de la vía Corduba-Emerita, que unía la capital de la Bética con la Lusitania.

-CIL.II, 4720: Miliario, al igual que el anterior, de Neron. Aunque le falta parte del texto, parece ser que este no difería en lo esencial del que aquí presentamos.

Apareció en el Campo de la Verdad (Córdoba), próximo al Arroyo de la Miel. Su paradero actual es desconocido. Por el lugar de aparición, es fácil que perteneciera a la Vía Augusta, en concreto al tramo comprendido entre Corduba y Ad Aras.

Existe un tercer epígrafe, en este caso se trata de un cipo en piedra molinaza, que apareció en la Dehesa de Alcorrucén, Pedro Abad (Córdoba), cuyo texto no presenta variaciones esenciales con respecto del nuestro. Por su lugar de aparición correspondería a la Vía Augusta en su tramo Ad Decumo y Epora.

No obstante, al no figurar en ninguno de ellos referencia alguna a cualquier tipo de obra realizada en la vía, parece ser que durante la época de Nerón se continuó la actividad constructora y reparadora de la Vía Augusta, comenzada durante el reinado de Claudio, su antecesor en el cargo.

Por último, cabe señalar, que desde el pasado día 11 de abril, y bajo el patrocinio de la empresa ENAGAS (4), la columna miliaria fue restaurada, en parte, e instalada para su contemplación en Sala IV del Museo Arqueológico Provincial de Córdoba (Lám. II).

(4) Las gestiones fueron realizadas por D. José Redondo, Ingeniero de ENAGAS, a quien desde aquí agradecemos todo su interés. 


\section{Bibliografia}

BATLLE HUGUET, Pedro (1946): Epigrafia Latina. C.S.I.C. Instituto “"Antonio de Nebrija"'.

CAGNAT, René (1964): Cours d' epigraphie latine. 4 Ed., "L'Erma”' di Bretschneider, Roma.

MELCHOR GIL, Enrique (1987): La red de comunicaciones romana en la provincia de Córdoba. Memoria de Licenciatura. Universidad de Córdoba. Inédita.

MELCHOR GIL, Enrique (1988): "La red viaria romana: El suroeste de la provincia de Córdoba”'. Ariadna, 4, págs. 28-42.

MELCHOR GIL, Enrique (1991): “Comunicaciones entre Astigi y la campiña de Córdoba, en época romana: Vía Augusta y camino de Metedores'. Ariadna, 8, págs. 6997.

PALli AGUILERA, Federico (1985): La vía augusta en Cataluña. Universidad Autónoma de Barcelona.

ROLDAN HERVAS, José Manuel (1975): Itineraria Hispana. Departamento de Historia Antigua, Universidad de Valladolid. Departamento de Historia Antigua, Universidad de Granada. 


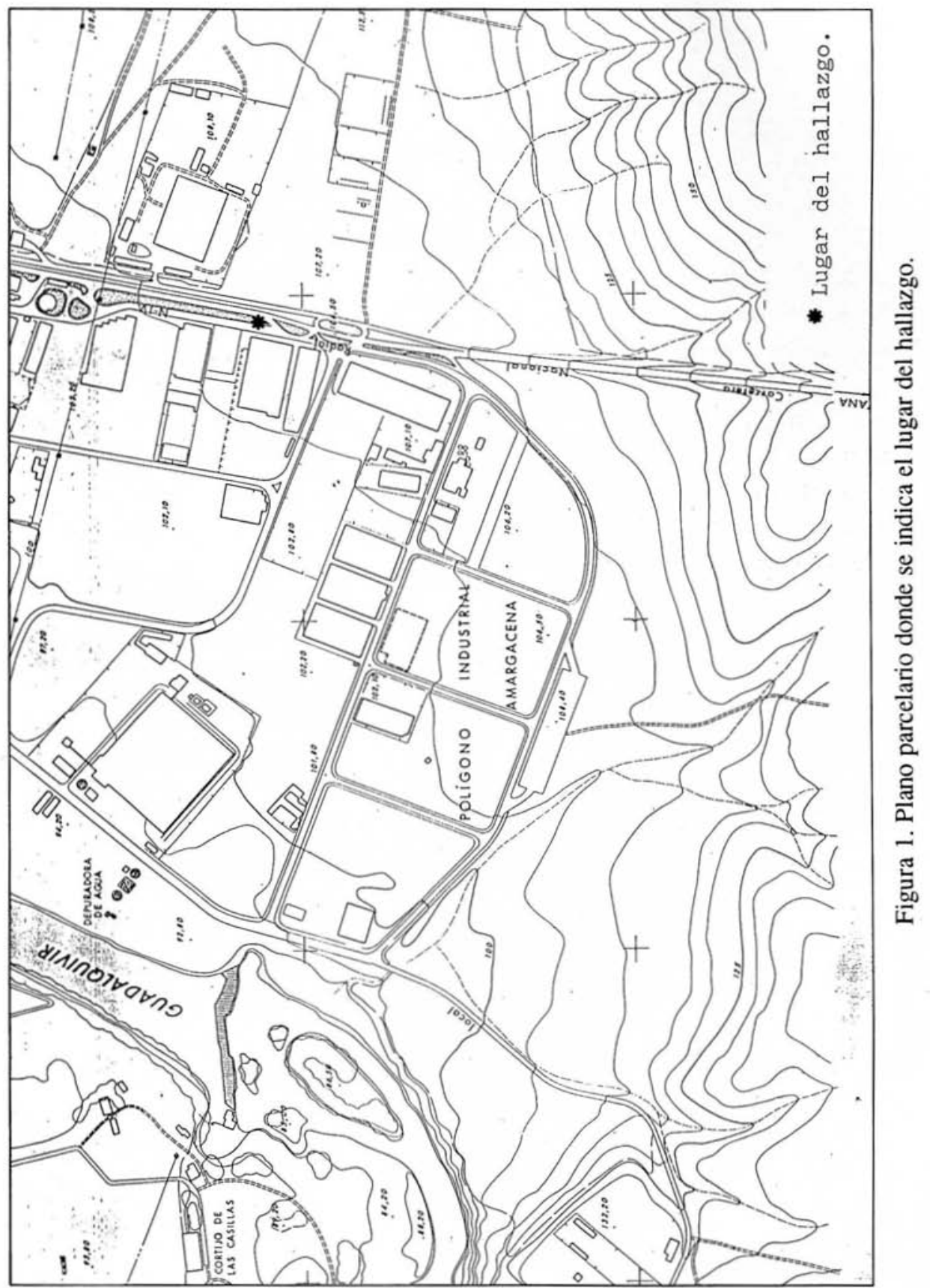




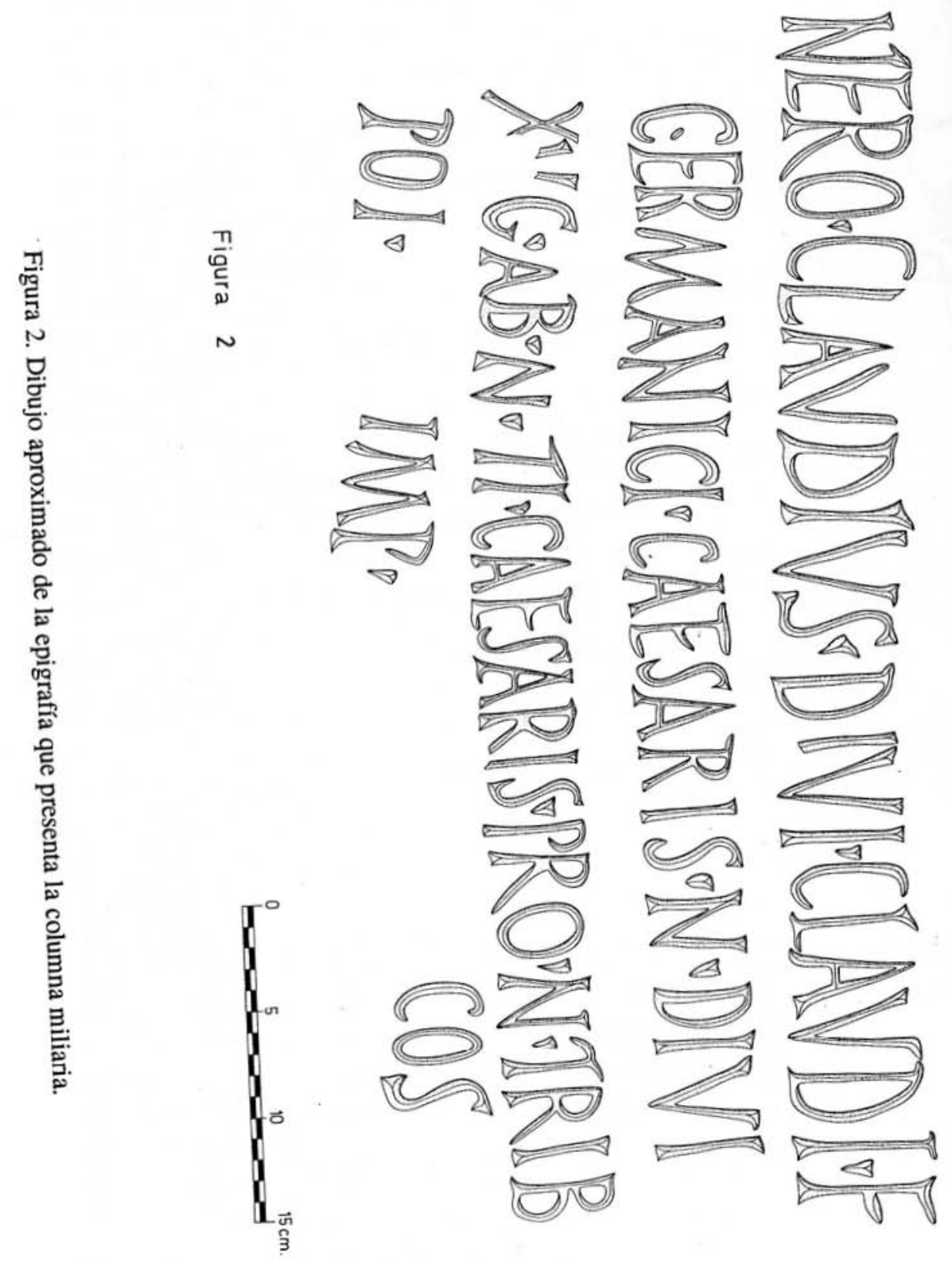




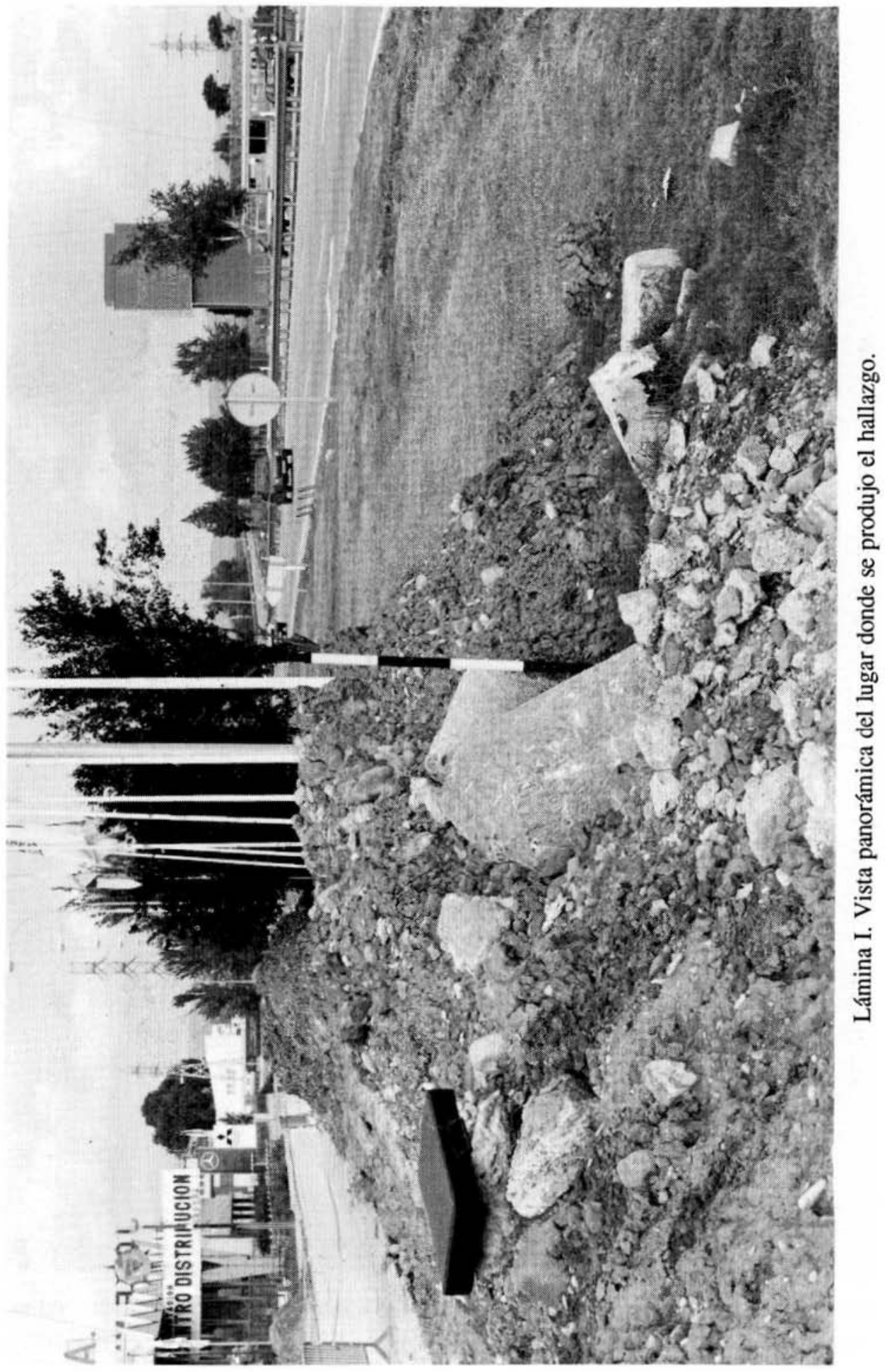




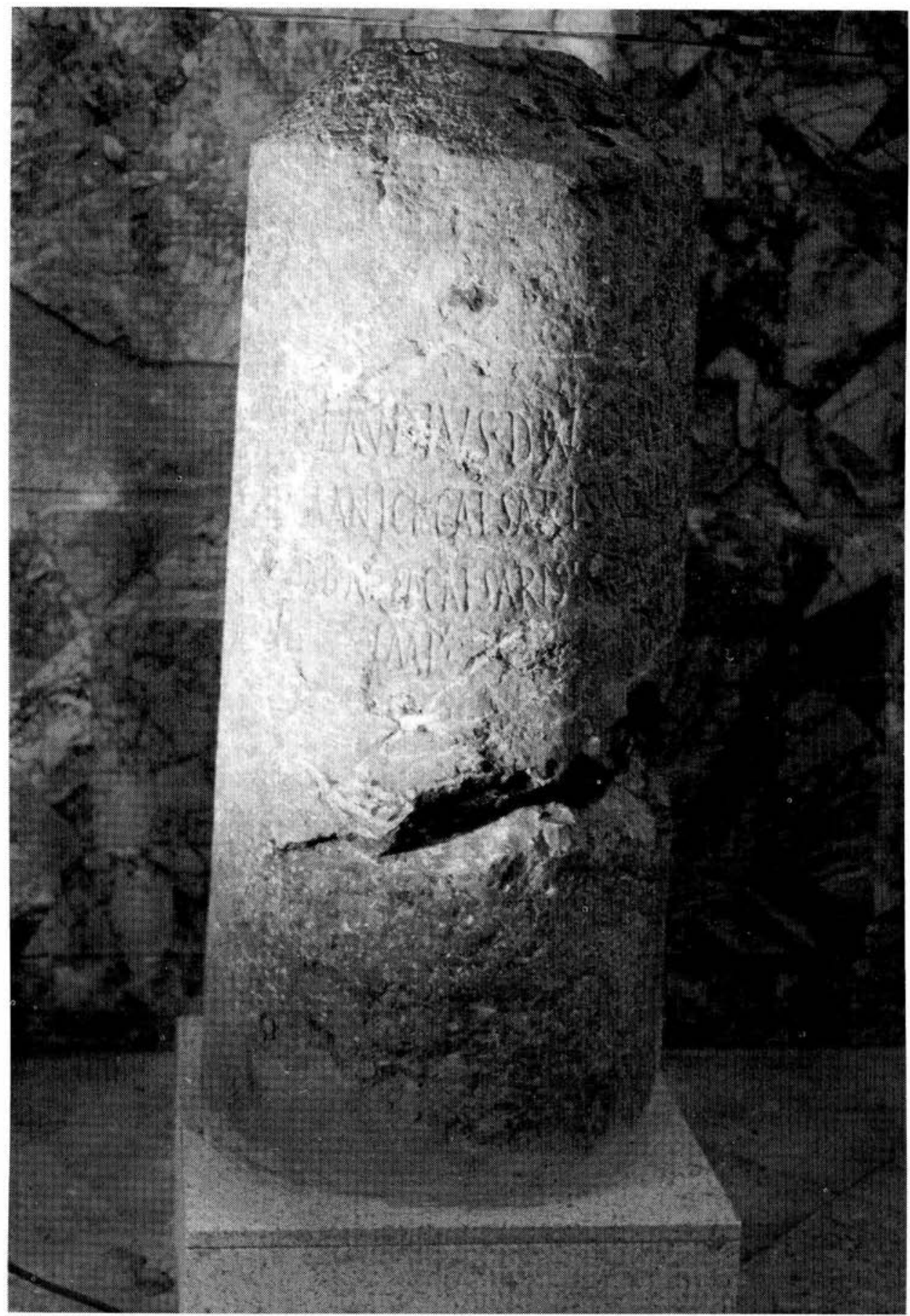

Lámina II. Columna miliaria, una vez restaurada e instalada en la Sala IV del Museo Arqueológico Provincial de Córdoba. 